\title{
PROYECTOS DE INVESTIGACIÓN Y EXTENSIÓN: IDENTIDADES, CIUDADANÍA Y FORMACIÓN DOCENTE ${ }^{1}$
}

\author{
Rita de Cássia PAIVA² \\ Universidade Federal do Pará
}

RESUMO: A UFPA - Universidade Federal do Pará - começou em 2009 com o PAPIM Programa de Apoio a Projetos de Intervenções Metodológicas - que busca incentivar e apoiar o desenvolvimento de atividades e experimentos que acrescente métodos e técnicas inovadoras e eficazes no processo de ensino e aprendizagem. A partir daí, foi desenvolvido o projeto Guamá Bilíngue de pesquisa, ensino e extensão que contempla diversos aspectos do processo de ensino de ELE, já que é uma proposta educacional centrada em uma metodologia diferenciada especificamente desenhada para alunos de ensino médio entre 15-18 anos em risco social de escolas públicas do bairro Guamá, Belém/PA, na Amazônia brasileira. O desenvolvimento do material, com seleção de textos para as unidades, alternadamente, mesclando temas culturais e sociais que afetam diretamente a vida de jovens em bairros periféricos, se fez possível usando estratégias da linguística cognitiva bem como o uso de analogias para compreensão do novo código linguístico que está sobrepondo-se ao seu próprio. Isto porque, segundo pesquisa de Almeida Filho (apud Neta, 2004), "entre as línguas românicas, o português e o espanhol são as que mantem maior afinidade" já que se constroem numa base de $85 \%$ de semelhança. Para tanto, foram utilizados os princípios de modelo mental de Lawson \& Lawson (1993) assim como as teorias da aprendizagem significativa de David Ausubel (apud Moreira \& Mansini, 1982) e premissas de Vygotsky (1991), entre outros. Ao longo de setes anos de existência, o Guamá Bilíngue foi objeto de mais que duas dezenas de trabalhos em diversas áreas da formação docente e contribui positivamente nas relações dos adolescentes atendidos tanto no âmbito académico quanto social, além disso, está fomentando dois novos projetos de maior abrangência em diversas áreas vulneráveis da capital.

Palavra-clave: Guamá Bilíngue; Projetos de pesquisa e extensão; Ensino de ELE; Formação docente.

RESUMEN: La UFPA -Universidad Federal de Pará- comenzó en 2009 con el PAPIM Programa de Apoyo a Proyectos de Intervenciones Metodológicas- que busca incentivar y apoyar el desarrollo de actividades y experimentos que acrescente métodos y técnicas innovadoras y eficaces en el proceso de enseñanza y aprendizaje. A partir de ahí, fue desarrollado el proyecto Guamá Bilingüe investigación, enseñanza y extensión que contempla diversos aspectos del proceso de enseñanza de ELE, ya que es una propuesta educacional centrada en una metodología diferenciada específicamente diseñada para alumnos de enseñanza media entre 15-18 años en riesgo social de escuelas públicas del barrio del Guamá, Belém/PA, en la amazonia brasileña. El desarrollo del material, con selección de textos para las unidades, alternadamente, mezclando temas culturales y sociales que afectan directamente la vida de jóvenes en barrios periféricos, se hizo posible usando estrategias de la lingüística cognitiva así como el uso de analogías para comprensión del nuevo código lingüístico que se está sobreponiendo al suyo propio. Esto porque, según investigación de Almeida Filho (apud Neta, 2004), "entre las lenguas románicas, el portugués y el español son las que mantienen mayor afinidad" ya que se construyen en una base del 85\% de semejanza. Para tanto, fueron utilizados los principios de modelado mental de Lawson \& Lawson (1993) así como las teorías del aprendizaje significativo de David Ausubel (apud Moreira \& Mansini, 1982) y premisas de

\footnotetext{
${ }^{1}$ Artículo producido con base en trabajos presentados por grupo de investigación, enseñanza y extensión Guamá Bilingüe: http://dgp.cnpq.br/dgp/espelhogrupo/5561497054806008.

${ }^{2}$ Formación doctoral en Estudios de la Traducción en la UFSC; profesora coordinadora del curso de Letras Español, en la Universidad Federal de Pará. Contacto: cassia@ufpa.br
} 
Vygotsky (1991), entre otros. A lo largo de siete años de existencia el Guamá Bilingüe ha sido objeto de más que dos decenas de trabajos en diversas áreas de la formación docente y contribuye positivamente en las relaciones de los adolescentes atendidos tanto en el ámbito académico cuanto social, además está fomentando dos nuevos proyectos de mayor abarcadura en diversas áreas vulnerables de la capital.

Palabras-clave: Guamá Bilingüe; Proyectos de investigación y extensión; Enseñanza de ELE; Formación docente.

\section{Introducción}

Uno de los tópicos más importantes actualmente es la cuestión educacional. Con las Metas 2021 de la Organización de Estados Iberoamericanos se tiene una perspectiva más abarcadora de los retos que se buscan vencer en Latinoamérica en el ámbito educacional. En las páginas de dichas Metas se menciona lo que se lee a continuación:

Una educación más justa exige mayor equidad social y mayor nivel cultural, aspiración que si bien se extiende a toda la ciudadanía, pretende orientarse especialmente hacia aquellos colectivos tantos años olvidados: los grupos originarios, los afro descendientes, las mujeres y las personas que viven en zonas rurales. En esta perspectiva se sitúa el compromiso de crear un fondo solidario para la cooperación educativa que complete el esfuerzo de los países y de las zonas más pobres de la región para lograr las metas acordadas. (2021 METAS EDUCATIVAS, 2010, p. 9-10)

De este modo, la educación ha cobrado un valor que le había sido denegado en los últimos años condicionada a mero aderezo en los programas gubernamentales, incluyendo ahora, la creación de un fondo que auspicie su consecución para las zonas más necesitadas de ayuda en Latinoamérica.

Es todo muy bonito si no fuera más que un arreglo cosmético para camuflar los fallos diversos que son inherentes al sistema educativo. En Brasil, por lo menos, se busca, sobre todo, el mero desarrollo curricular más bien la repetición de los saberes históricamente construidos por la hegemonía que conduce al mantenimiento del sistema educacional con los conocimientos formales valorados sobre la construcción holística del alumno. Más ahora en que la Medida Provisoria (MP) 746/201 y la PEC $^{3} 55$ han sido aprobadas sustrayendo materias como sociología, educación física, lengua española ${ }^{4}$ entre otras, de la enseñanza media. Lo peor es que dichas alteraciones permiten que personas que no tiene la carrera docente puedan actuar en clase.

Además, la estructura escolar, heredada de la revolución industrial cuya perspectiva era formar mano de obra sumisa, no abarca el aspecto de formar ciudadanas y ciudadanos que serán capaces de romper con los ciclos jerarquizados a que la educación está sometida o tampoco permitir que el libre pensamiento pueda adueñarse de sus alumnos. Se sabe que alumnos originarios de las escuelas públicas brasileñas sufren de un desfasamiento con relación a los contenidos enseñados así como a los procesos cognitivos que deben ser desarrollados en la educación formal. Así, los proyectos Guamá Bilingüe, Formación Ciudadana en E/LE y Lengua, Cultura y Ciudadanía intentan llevarles una alternativa de formación para alumnos de la secundaria de escuelas públicas que los envían para aprender español.

\footnotetext{
${ }^{3}$ Propuesta de Enmienda Constitucional

${ }^{4}$ En la contra la Ley 11.165 de 05 de agosto de 2010.
} 
Cabe decir que, de un modo general, a pesar de los innúmeros esfuerzos de los gobiernos, siquiera la mitad de las escuelas públicas de Brasil cuentan con infraestructura básica tales como aulas con paredes, puertas, ventanas, capacidad eléctrica para enchufes y bombillas, ventilador o aire acondicionado, además de pizarra, mesa y silla para los profesores y pupitres para todos los alumnos.

\section{Los proyectos}

Tratar, entonces, de alternativas educacionales para comunidades de alumnos en entornos de violencia y riesgo social se convierte en una más de las utopías que emergen desde la clase docente. No obstante, han sido desarrollados en Belém, capital del estado de Pará, en la Amazonía brasileña, un proyecto de investigación en educación con actuación extensionista que trata justamente de intentar llevar los/las estudiantes a un mejor porvenir en sus estrechos horizontes académicos. El proyecto Guamá Bilingüe nació el 2010 del intento de integrar la Universidad Federal de Pará -UFPA- al barrio del Guamá -el más grande y uno de los más violentos de la ciudad. A partir del 2016, dos otro proyectos nacieron basados en la misma metodología. Uno, Formación ciudadana en E/LE, es igualmente auspiciado por la Pro-rectoría de Enseñanza. El otro, Lengua, cultura y ciudadanía, es patrocinado por la Pro-rectoría de Extensión.

Para participar de convocatorias que aportaran recursos para su ejecución, tres proyectos fueron creados. El Guamá Bilingüe se presenta, desde el 2009 al PAPIM -Programa de Apoyo a Proyectos de Intervenciones Metodológicas- que anualmente busca incentivar y apoyar el desarrollo de actividades y experiencias que propague métodos y técnicas innovadoras y eficaces en el proceso de enseñanza y aprendizaje. Para el PROINT -Programa Integrado de Apoyo a la Enseñanza, Pesquisa y Extensión- se presentó el proyecto Formación Ciudadana en E/LE. Para la convocatoria del PIBEXT -Programa Institucional de Becas de Extensión- se presentó el Lengua, Cultura y Ciudadanía.

Hay que señalar que estos subsidios son necesarios para alcanzar el ánimo de atención social de la UFPA cuyos dos tercios están insertos dentro del barrio Guamá y el otro tercio en Terra Firme. Ambos barrios son zonas de peligro para los residentes y más para los jóvenes. En el texto del proyecto se menciona que:

Este barrio es, reconocidamente, uno de los más violentos de esta capital debido a las desigualdades sociales y a los parcos recursos en él invertidos por los sectores públicos y privados para la mitigación de esta situación.

Siendo la UFPA una institución pública con una fuerte inclinación social en sus diversos cursos, está inserta en una relación de amensalismo ${ }^{5}$ con su entorno que le impide alcanzar su plenitud académica.

Partiendo de esa premisa, creemos que la educación -como agente transformador- puede iniciar un ciclo de cambios positivos que culminen en una relación más productiva para ambos, a saber, UFPA y barrio del Guamá. (GUAMÁ BILINGÜE, 2010)

Se sabe que desde la década de los 80 del siglo XX se usa la metodología comunicativa exitosamente para enseñanza de lenguas extranjeras. Hasta aquel entonces otros métodos eran

\footnotetext{
${ }^{5}$ Interacción biológica que se produce cuando un organismo se ve perjudicado en la relación y el otro no experimenta ninguna alteración.
} 
utilizados en Brasil. En términos de español, el primer material didáctico para E/LE fue el manual de Idel Becker de 1945. A partir de ahí, varios otros métodos llegaron al mercado hasta que el método comunicativo se consolidó como el más efectivo.

Para cuestiones de aprendizaje, hay que considerar, en términos de la relación portugués/español y viceversa, la presuposición del saber (CELADA, 2000, p. 37) que hay entre hablantes de los dos susodichos idiomas. En otras palabras, los hablantes de cada idioma piensan que su propio conocimiento del idioma del otro es suficiente para alcanzar sus propósitos comunicativos. Lo que no es verdad en definitiva.

Si por un lado la fonética del español es más simple para el alumno brasileño que viceversa, la musicalidad del español es de difícil reproducción para aquél. Para que se adquiera un nuevo sistema lingüístico es necesaria la instalación de nuevos conceptos y de mensajes organizados a partir de elementos fonéticos, léxicos, semánticos y sintácticos -por ejemplo. Estos nuevos conceptos y mensajes son diferentes y arbitrarios (LANCHEC, 1976, p. 35) como suelen ser los signos lingüísticos. La adquisición misma implica en un nuevo tipo de decodificación entre significantes y significados. Aunque semejantes, cada uno de los dos idiomas es aquel mismo sistema abstracto de signos interrelacionados, de naturaleza social y psíquica, obligatorio para todos los miembros de una comunidad lingüística definida en los términos de Saussure. Así que, suposición del saber no implica en saber de hecho el otro idioma.

Aun así la enseñanza de E/LE, por su gran similitud al portugués, puede ser hecha por un camino abreviado: el análogo. Por lo tanto, a partir de esta perspectiva se propuso la inserción del pensamiento analógico como parte de una metodología distinta a fin de haber aprendizaje significativo para los alumnos que son un público bastante específico en un tiempo más corto.

Para entender el ámbito y alcance del proyecto Guamá Bilingüe y sus renuevos, se debe decir que la Universidad Federal de Pará -UFPA- es la mayor institución de enseñanza en la pan Amazonía, con más de 30 mil estudiantes. Es, al contrario de otras IFES ${ }^{6}$, una universidad multi-campus y está presente en 10 ciudades del interior del estado de Pará, además del Campus Guamá, en la capital, Belém, ubicación de los proyectos aquí presentados. Por ello, se percibe la necesidad de aportar a la comunidad en la que está UFPA proyectos de extensión ya que la Universidad se apoya en el trípode enseñanza, pesquisa y extensión en el ámbito académico. Si se considera el valor anual para la dotación de cada proyecto, se tiene un monto por alumno máximo al año de $120 \mathrm{\epsilon}$, lo que significa unos $1.200 \mathbf{\epsilon}$, lo que los convierte el proyecto en uno de los más baratos del país.

Así, a partir de una iniciativa de la propia Universidad, a través de una convocatoria del 2009, tiene su inicio en el año 2010 el Guamá Bilingüe, que atiende en dos grupos de 15, a alumnos de dos escuelas públicas del barrio Guamá, que tengan entre 15 y 18 años y estén en riesgo social. Cabe informar que, aunque el proyecto esté en su séptimo año, es sometido cada convocatoria y, hasta ahora, ha sido evaluado e aprobado para seguir actuando.

Con el éxito del Guamá Bilingüe, en el grupo de investigación hubo una decisión colectiva de intentar ampliar la abarcadura del proyecto. Así, fueron sometidos a dos otras convocatorias en octubre del 2015 los proyectos Formación Ciudadana en E/LE y Lengua, Cultura y Ciudadanía. La dotación presupuestaria del primero es la misma del Guamá Bilingüe, sin embargo permite que haya cuatro grupos con cerca de 25 de alumnos cada uno. El segundo, tiene perfil más abarcador en el momento que permite diez voluntarios que hacen turnos para enseñar en cinco zonas de las ciudades de Belém y Ananindeua con cerca de 15 alumnos cada uno. Tanto el

\footnotetext{
${ }^{6}$ Instituições Federais de Ensino Superior
} 
Guamá Bilingüe cuanto el Formación Ciudadana en E/LE tienen becarios para actuar como profesores, ya Lengua, Cultura y Ciudadanía permite que los voluntarios (diez registrados en el proyecto) actúen también como profesores; el becario trabaja como asesor de la coordinación.

\section{Justificante}

Para la realización del proyecto como una intervención metodológica original, fue necesario considerar que los procesos mentales para la adquisición de una lengua extranjera pasan también por el razonamiento analógico. Méier (1974 citado en Paiva, 2008, p. 3284) ilustra que:

La adquisición de otra lengua implica la instalación de un nuevo código lingüístico, un nuevo sistema de codificación y decodificación, independiente del sistema utilizado hasta entonces. Podemos imaginar que esos dos sistemas se desarrollen o independientemente uno del otro, si la realidad cubierta por los significados es diferente, o en superposición, si los significados son interpretados sucesivamente en las dos lenguas.

Los autores Shank y Cleary (1995, p. 73) enfatizan que, en la adquisición de nuevos conocimientos, es necesario que determinados criterios validen el conocimiento involucrado en el proceso enseñanza-aprendizaje que extrapolan verificación y presuponen comprensión, verdad-veracidad y rectitud:

Respecto a los criterios de validación, lo que hace que la comunicación se pueda conseguir sola y por si: lo que se está diciendo es comprensible (inteligibilidad); que las afirmaciones que se hacen son ciertas (verdad); que lo que se está diciendo encaja en el contexto y está justificado (veracidad) y que quien habla es sincero e no pretende engañar (rectitud). Criterios cuyo alcance es infinitamente más amplio que la verificación científica. Y, por lo tanto, resultan más adecuados para el tratamiento de los valores en educación ${ }^{7}$.

Y, tratándose de valores en educación para un público de jóvenes en riesgo social, diversos cuidados fueron tomados, desde la sumisión misma del proyecto PAPIM a la "Pro-rectoría de Ensino e Graduação" de la UFPA el 2009 hasta la elección de textos y montaje de los materiales.

Se consideró también, el punto de vista de Mondoñedo (2008, p. 9)

[...] el ejercicio normativo no es una praxis para conocer, buscar y comunicar la verdad, no es un ejercicio científico, sino una acción desarrollada para ocultar la heterogeneidad y la riqueza de la expresión verbal, una estrategia de la ilusión con explícitas finalidades de control; en otras palabras, es un instrumento ideológico.

y se decidió la elaboración de un material poco gramatical, menos aún doctrinario y sobretodo que pudiera abrirles la mente y permitirles una perspectiva diferente de una vida de restricciones y estrecho porvenir a los alumnos participantes en el proyecto.

Para que se pueda mensurar la educación pública en Brasil y en el ámbito de las perspectivas de jóvenes como los de los proyectos, aquí se exhiben datos del Instituto de Co-

\footnotetext{
7 Traducido del inglés.
} 
Responsabilidade pela Educação (Magalhães, 2010) para alumnos originarios de las escuelas públicas de Brasil

1. Alta tasa de fracaso y de abandono escolar.

2. 13 años para cumplir el ciclo fundamental de 9 años.

3. 17 años para cumplir el ciclo básico de 12 años.

4. Solamente el $15 \%$ de los alumnos concluyen la enseñanza fundamental con edad correcta (14 años).

5. Solamente el $40 \%$ de los jóvenes brasileños concluyen la secundaria.

6. El $50 \%$ de los alumnos del $5^{\circ}$ curso son analfabetos.

7. Los alumnos concluyen el $9^{\circ}$ curso con nivel de conocimientos del $5^{\circ}$ curso.

8. Los alumnos concluyen la secundaria con nivel de conocimientos del $9^{\circ}$ curso.

9. El 70\% de la población brasileña es analfabeta funcional.

10.El $80 \%$ de la población brasileña no consigue calcular un porcentaje.

Claro está, por lo tanto, que no sirve entregar a los alumnos un material que no tenga conexión y/o referencia a su entorno, a su contexto, a su realidad. En otras palabras, ese público necesita un material que les conduzca al aprendizaje significativo (AUSUBEL citado en MOREIRA \& MANSINI, 1982), para que haya construcción de conocimiento. El aprendizaje significativo ocurre cuando la nueva información se ancla en conceptos relevantes prexistentes en la estructura cognitiva de quien aprende.

La adquisición de conceptos por medio del aprendizaje receptivo no es solo un proceso pasivo de abstracción. A pesar de no ser del mismo tipo del de la formación de conceptos, es básicamente caracterizada por un proceso activo de interacción con los conceptos ya adquiridos. Cuanto más activo sea este proceso, más significativos y útiles serán los conceptos. Y para que sea posible hacer más activo el proceso este, es fundamental que interactúe profesor-alumno con el método y con los alumnos del proyecto.

\section{Materiales y método}

Debido a restricciones presupuestarias, el material utilizado fue pensado para ser de bajo costo $\mathrm{y}$, ya que los materiales didácticos [libros] existentes en el mercado no contemplarían totalmente la filosofía del proyecto que era, además de enseñar Español, discutir transformaciones cambios y mejoría de condición de vida a través del estudio y también tutelar problemas sociales que pudieran estar encadenados a las vivencias de los alumnos, se decidió por la creación y montaje del material para el proyecto.

Todo ello se dio porque la labor de enseñar español a estudiantes brasileños es muy ardua. Sobre todo cuando se trata de enseñar español a adolescentes de 15 a 18 años en riesgo social de uno de los más violentos barrios de la ciudad de Belém/PA. Así que, para que existieran los proyectos se hizo necesario el uso de estrategias de la lingüística cognitiva así como el de analogías para comprensión del nuevo código lingüístico que se está sobreponiendo al suyo propio, crear una metodología específica para atender a aquel público específico. Si no hubiera sido de esta manera, los materiales serían más bien "español a través de textos" sin más repercusiones en la sociedad.

En el afán de aportar una alternativa diferente a jóvenes que estudian en la red pública alrededor de la UFPA, fueron seleccionados 30 alumnos de dos escuelas públicas del barrio de Guamá, en Belém, capital del estado de Pará, en la Amazonía brasileña, como 'proyecto piloto' en 2010, 
35 alumnos de las mismas escuelas para los años 2011 y 2012 y 38 alumnos el 2013. En 2014, estaban suscritos 48 alumnos de los cuales 42 terminaron. El 2015 fueron 32 alumnos y 23 concluyeron. El 2016 los tres proyectos cuentan con cerca de 200 alumnos participantes.

Se tomó en cuenta la necesidad de alejar a los alumnos de las actividades callejeras cotidianas que están comprendidas desde cotilleos en las esquinas a pequeños robos e ilegalidades como llevar de arma de fuego. En los dos primeros años, para la selección de los participantes se contó con el inestimable apoyo de las directoras de los dos institutos de donde salieron los alumnos que fueron elegidos en el período vespertino porque los alumnos del matutino son jóvenes de "familia" que no están por la calle y los del nocturno trabajan todo el día, en opinión de dichas directoras.

Sin embargo, ha habido algunos cambios en el público. Los alumnos, a partir del 2012, ya no estaban divididos de aquella manera. Por diversas contingencias sobre todo por las innumerables huelgas de la red pública en Belém y la falta organizacional de la gestión pública, se hizo necesaria la adaptación de los alumnos en las escuelas para adecuar un nuevo estilo de alumnos, que ya no estaban más separados conforme sus perfiles. Con ello, para acoger estos jóvenes con sus idiosincrasias, el proyecto tuvo que ser adaptado y, de la misma manera, hacer nuevos entrenamientos con los becarios.

Para abarcar tamaño ámbito, fue necesario construir un material único con un abordaje metodológico exclusivo que les permitiera a los alumnos no solamente el aprendizaje de español sino también el acceso al conocimiento del mundo que les falta. Todo proceso creativo fue pautado en el uso de vocablos con mucha proximidad al portugués, palabras transparentes y se evitó el uso de estructuras y vocabulario que pudiera sugerir palabrotas o expresiones soeces en portugués.

En el ámbito del léxico, se adaptaron los textos para utilizar palabras transparentes pero con vocabulario no semejante entre portugués y español se buscaron estructuras que llevaran a la comprensión contextual. Por ejemplo:

Comprei uma ........ de vinho para .......

Compré una botella de vinØo para regalar

La cuestión fonética que se trabajó en la primera unidad, antes del texto, se convirtió en el resorte de impulsión para el habla. Las primeras clases fueron convertidas en un taller de corrección fonética. Esto porque era necesario que los alumnos tuvieran seguridad para leer en voz alta y, a su turno, conversar en los diversos momentos a eso destinados. A continuación, vino la parte de formación de la lengua y cultura hispanófonas. Al fusionar la enseñanza de la lengua con la cultura que la había generado, el nivel de interés aumentó significativamente: había ya más comprensión y ya no era necesario controlar tanto las situaciones comportamentales. La parte entretenida fue presentar un dibujo animado "El camino hacia El Dorado" en el que el doblaje 'mejicano' de la película funcionó como un eslabón comunicativo muy importante. Hubo diversas discusiones sobre, incluso, la elección de vocabulario, la sinonimia y prosodia en las que los alumnos se involucraron.

Ya en el aspecto gramatical, las explicaciones trataron de hacer un paralelo con el conocimiento de la lengua materna que ellos ya habían estudiado. No fue de hecho una sorpresa descubrir que el $60 \%$ de los alumnos no sabían siquiera el conocimiento básico de conjugación adecuada dos verbos en portugués. Cabe señalar que ese punto no era estudiado estructuralmente, sino en el contexto de la unidad. Así que, al fin y al cabo, muchos alumnos aprendieron en español lo que no habían aprendido en portugués. 
Fue necesario un cuidado especial con el léxico y cada texto escrito alternaba por unidad didáctica algo de la realidad de los alumnos - como alcoholismo, violencia doméstica y acoso escolar- con informaciones culturales -como El Cid, Joaquín Murieta, Mercedes Sosa y Patagonia. Cada una de las unidades tiene sólo cuatro páginas y compuestas por un texto, diez preguntas de interpretación sobre él; una sección para discusión proponiendo la fluidez linguística y participación individual de cada alumno denominada "Hablar por los codos" en la que los alumnos tienen que hablar y expresar sus pensamientos y percepciones sobre cada tema presentado; una sesión de gramática con ejercicios "¡Atar cabos!” y "Poniendo las manos en la masa" respectivamente; "¡Fíjate”! con un complemento cultural o social y terminando con "Recordar".

Hay que recordar que la elaboración de los materiales se dio básicamente sobre dos premisas: la del aprendizaje significativo y del uso de las analogías para acortar el camino entre lo que ya se sabe y lo que se espera que los alumnos aprendan. Es decir, con la configuración de sobreponer los conocimientos a través del pensamiento analógico y utilizando análogos estructurales, léxicos y semánticos interlinguales, se llega al aprendizaje significativo y al mejor manejo de los contenidos aprendidos por los alumnos; así fue delineado el enfoque metodológico para la creación del material. Los por textos y ejercicios igualmente salieron de webs de periódicos y otras ubicaciones informáticas que nos permitieran elaborarlos sin infringir los derechos de autor.

Para que esto fuera posible, el acceso a textos y estudios se hizo con el uso de internet en búsqueda en sitios web que fueran de libre uso de sus contenidos como los "Creative Commons", que tienen como objetivo apoyar y construir un dominio público más amplio en el que las obras puedan ser vehiculadas sin herir derechos de autor. Los llamados "cc" miran también permitir manejar la cultura de una manera más libre. De toda manera, los materiales elaborados están disponibles a quienesquiera utilizarlo.

A continuación se tiene el esquema de las unidades:

1. Bienvenidos al español: Bienvenida. Presentación del proyecto, metodología y personal involucrados; clase de fonética $y$, además de explicaciones sobre el proceso de pensar 'analógicamente'.

2. Unidad 1: ¿Qué estoy haciendo aquí? Discusión sobre el cambio a través del esfuerzo y estudio con relato de dos alunas oriundas de proyecto social semejante.

3. Unidad 2: Acoso escolar. Análisis sobre la violencia escolar, causas, consecuencias y formas de evitarla.

4. Unidad 3: Joaquín Murieta. La historia de aquel que puede haber sido el Zorro, sirve para que se comprenda la necesidad de que seamos responsables por nuestras actitudes.

5. Unidad 4: Tener Clase. Esa unidad se propone discutir la ética cotidiana así como cuestionar la corrupción y también se hacen discusiones sobre modos de evitarla.

6. Unidad 5: $\mathrm{El} \mathrm{Cid}$. El legendario héroe español es tema sobre la necesidad de luchar por ideales y lo que se considera justo a pesar de las adversidades.

7. Unidad 6: Alcoholismo adolescente. Discute la problemática de la presencia del alcohol en las relaciones de los adolescentes, así como las consecuencias de su ingestión.

8. Unidad 7: El Derecho. Introducción al tema con presentación de parte de la Constitución Federal y de la Declaración de los Derechos Humanos del 1789; orienta los alumnos sobre sus derechos/deberes como ciudadanos.

9. Unidad 8: ¿Cultura o tortura? Aborda dos temas polémicos: las corridas de toro en España y el ritual ceremonial del Tinku en Bolivia; en ambos la 
violencia es inmensa, en el primero con el toro y en el segundo en grupos de edad específicos.

10.Unidad 9: ¿Qué se entiende por violencia basada en el género? Retrata la triste exposición de la violencia doméstica en contra la mujer, hecho muy corriente en la realidad de los alumnos del proyecto según los propios alumnos. En esta unidad se escuchan dos canciones, una de España y otra de Guatemala sobre. Ambas canciones tratan de la violencia en contra la mujer. 11.Unidad 10: Patagonia. Trata del calentamiento global y, por esta ser la última unidad debido a las antedichas huelgas, trae como último tópico un cuestionamiento sobre las enfermedades sexualmente transmisibles tan presentes en la vida de esos adolescentes. (PAIVA \& LEAL, 2011, p. 10981099).

En el ámbito del proyecto están descritas trece unidades didácticas. Por los motivos ya señalados, solamente se ha podido aplicar solamente las diez explicadas anteriormente. Sin embargo, ya están proyectadas las tres unidades subsecuentes: la Unidad 11 tratará de la unión entre iguales, la Unidad 12, de la literatura y la Unidad 13 sobre el porvenir, a fin de instar los alumnos de los proyectos a buscar posibilidades prometedoras apara sus vidas.

El proceso no es solamente usar de los textos sino acercar los becarios, voluntarios y alumnos de una conciencia que ayude a todos los involucrados a considerar la realidad a que están sometido. Además de fomentar en los alumnos la literacidad crítica, utiliza el pensamiento analógico que acorta el proceso de aprendizaje y, en su constitución, considera que la analogía tiene un rol tan central en el aprendizaje, en la memoria y en el raciocinio (Lawson y Lawson, 1993, p. 2). La metodología de enseñanza está totalmente centrada en el profesor, es decir, la intervención de los profesores-alumnos (becarios) es lo que conduce los alumnos del proyecto a que no solo aprendan, sino empiecen a despertar epistemológicamente, a hacer las interacciones necesarias para que ocurra el aprendizaje significativo y sobretodo, pasen a tener más percepción de su entorno y caminen hacia la real ciudadanía.

\section{La formación docente}

Las carreras de formación docente en Brasil tienen un escoplo bastante amplio en sus currículos. Sin embargo, en la UFPA, hay solamente 408 horas de prácticas divididas únicamente en los dos últimos semestres. Esto conlleva el problema de que en los siete semestres anteriores los alumnos estudian teoría y conocimientos específicos de lengua, pero desconocen la realidad escolar.

En las clases y en las prácticas mucho se les muestra, pero el contacto directo con alumnos reales en situaciones cotidianas de escuela solo existen tras tres años y medio de estudios. Así, en el anhelo de ofrecer también a los estudiantes de la carrera de Letras Español, fue creado el 2013 y debidamente registrado en el Consejo Nacional de Desarrollo Científico y Tecnológico $-\mathrm{CNPq}^{8}$ el grupo de investigación Guama Bilingüe ${ }^{9}$. Dicho grupo consta de 13 investigadores, 19 estudiantes y 10 voluntarios.

Para que la actuación sea conforme las propuestas, son hechas reuniones semanales, alternando una pedagógica y una general, esta para discusión de presentación de trabajos, actualización de materiales y discusiones diversas. Las pedagógicas tratan de la pedagogía y la educación,

\footnotetext{
${ }^{8}$ Conselho Nacional de Desenvolvimento Científico e Tecnológico en portugués.

${ }^{9}$ http://dgp.cnpq.br/dgp/espelhogrupo/5561497054806008
} 
actitudes que deben tener los profesores hacia los estudiantes, etc. En ambas reuniones también se presentan los temas de las unidades didácticas, como impartirlos, como conducir los estudiantes al cuestionamiento de su realidad y perspectivas de cambio futuras.

Para los alumnos de la carrera de español esta es la oportunidad de participar de un proyecto de investigación, enseñanza y extensión al mismo tiempo que es el primer contacto con un ambiente real de enseñanza. También les permite discutir cuestiones relativas a su formación y a los retos que enfrenta un docente en la región de la amazonia y pueden utilizarse del proyecto como laboratorio de investigación y aplicación de sus trabajos académicos.

De esta manera, cuando los alumnos quieren implementar alguna actividad relacionada a las materias del curso, ellos presentan la propuesta al grupo y se la analiza en términos de asunto (léxico, temático, gramatical, etc.) y, si es aprobada, se agenda su aplicación bajo la supervisión de un investigador. Dependiendo de la calidad del trabajo, se lo puede incorporar como material complementario a las unidades.

\section{Conclusión}

Parcialmente, se puede decir que los resultados son positivos debido al creciente interés por parte de los alumnos para aprender el idioma español y por las pequeñas modificaciones positivas de actitud percibidas hasta el modo que los alumnos se tratan entre ellos. El equipo cree que, a medida que las clases y demás actividades son desarrolladas, hay más interés y compromiso de los alumnos.

Durante los tres primeros años del proyecto, diversos alumnos del Guamá Bilingüe han intentado, sin éxito, aprobar las selectividades de las universidades en Belém. A cada año en las reuniones del grupo de investigación que nortea los trabajos del proyecto intenta mejorar los materiales y su enfoque para aumentarles las posibilidades a los alumnos participantes. Claro está que cada grupo es diferente del otro, pero algunos estándares de actitud y de aprendizaje ya están siendo observados y, para los grupos que entran a partir del 2014, se consideró como marco de psicología del aprendizaje. Cabe informar que, por primera vez, dos alumnos del Proyecto del año 2013 fueron aprobados en la selectividad para la UFPA Universidad Federal de Pará-: uno para la carrera de sistemas informáticos y la otra para la carrera de nutrición. A partir de ahí, varios otros que intentaron han ingresado en las diversas universidades de la región, tanto públicas como privadas.

Se puede decir que enseñar español a estudiantes brasileños a través de raciocinio analógico no sólo es posible sino deseable. Además de hacer más fácil la labor del profesor, les auxilia a los alumnos a buscar más informaciones por ellos mismos y a alcanzar autonomía en el proceso de aprendizaje. Logros así son aún más significativos para un público tan específico como adolescentes en riesgo social que han visto en el aprendizaje de español una puerta para su acenso socio académico y también para ingresar en el mercado laboral. Así, aquellos alumnos que miraban a los profesores del proyecto de reojo con la desconfianza de los rechazados sociales ya empiezan a buscar en una biblioteca o en la internet materiales en español en vez de estar por las calles buscando 'distracciones' como la estafa, el crimen y la droga.

Diversos logros están siendo obtenidos y son aún más expresivos para un público tan específico como adolescentes en riesgo social que empiezan a buscar en un cibercafé materiales en español en vez de 'distracciones' como la estafa. Además, de enseñar español, el Guamá Bilingüe está 
convirtiéndose en un instrumento de cambio personal y educacional tanto para os alumnos del proyecto como para los becarios que en él actúan.

\section{Referencias bibliográficas}

CELADA, M. T.; GONZÁleZ, N. M. Los Estudios de la Lengua Española. Anuario brasileño de estudios hispánicos -Suplemento El Hispanismo en Brasil. Embajada de España en Brasil; Thesaurus Editora, p. 35-58, 2000.

GUAMÁ BILINGÜE. Disponible http://www.ufpa.br/beiradorio/novo/index.php/2010/116-edicao-86setembro/1084-guamabilingue-em-10-anos http://www.sisprol.ufpa.br/view/projeto/index.php?view=projeto\&action=consultarStatus Accedido en 26 de diciembre de 2011 a las 11:25.

LANCHEC, J. Y. Psico-pedagogia e pedagogía das línguas. Rio de janeiro: Zahar Editores, 1976.

LAWSON D. I. \& LAWSON A. E. Neural Principles of Memory and a Neural Theory of Analogical Insight. Journal of Research in Science Teaching, v30 n10 p1327-48/Dec 1993.

MAGALHÃES, M. A. Transições e Transformações nas Relações Internacionais Acadêmicas: Novos Tempos, Novos Atores. Palestra de abertura da XXII REUNIÃO ANUAL DO FAUBAI, São Paulo, 14 de abril de 2010.

MÉIER, H. Ensaios de filologia românica. Rio de Janeiro: Grifo, 1974 (Coleção Litera 4).

MONDOÑEDO, M. R. Una ideología de la lengua: lo "correcto" y lo "incorrecto". Indiana: Universidad de Indiana Publisher, 2008.

MOREIRA, M. A.; MASINI, E. F. S. Aprendizagem significativa: A teoria de David Ausubel. São Paulo: Moraes, 1982.

NETA, N. F. A. Aprender español es fácil porque hablo portugués: ventajas y desventajas de los brasileños para aprender español. Recife: Universidade Federal de Pernambuco/ Cuadernos Cervantes, 2004.

Organización de Estados Iberoamericanos para la Educación, la Ciencia y la Cultura (OEI). 2021 Metas Educativas. Madrid: Cudipal, 2010. Disponible en www.oei.es/metas2021/sintesis.pdf Accedido en 17 de mayo de 2013 a las 14:58.

PAIVA, R. C.; LEAL, A. M. M. Projeto Guamá Bilíngue. In: IIII Congresso Internacional de Estudos Linguísticos e Literários na Amazônia, 2011, Belém. Anais... Belém: Editora CRV, 2011a, p. 1097-1100.

PAIVA, R. C. O espanhol no ensino cotidiano. In: I Congresso Internacional Da Associação Brasileira de Hispanistas e V Congresso Brasileiro de Hispanistas, Belo Horizonte. Anais... Belo Horizonte: UFMG, 2008 p. 3282-3291. 
SCHANK, R.; CLEARY, C. (1995). Engines for education. Hillsdale, New Jersey: Lawrence Erlbaum Associates, 1995. 
